\title{
breve historia de la fábrica uruguaya de neumáticos S.a. en el uruguay (1935-1974) short history of the fábrica uruguaya de neumáticos S.a. in uruguay (1935-1974)
}

\author{
Sebastián Sabini Giannechini $\star \star$ \\ Facultad de Ciencias Sociales, Universidad de la República, Montevideo, Uruguay
}

\section{RESUMO}

Este artigo busca, a partir da História de Empresas e Empresários, e sua variedade de visões, analisar o caso da Fábrica Uruguaya de Neumáticos S.A. (FUNSA), processadora de borracha e produtos de borracha, no período 1935-1974, que domina o mercado uruguaio naquele mercado nesse período. A fundação com capitais nacionais, seu desenvolvimento explosivo e diversificação produtiva baseada na proteção legislativa, estagnação e recuperação servem como um caso emblemático da indústria local e representam de maneira sintética o caminho seguido por ela. Paralelamente, são realizadas diversas estimativas do Valor Adicionado Bruto, Ocupação e Salários da empresa e do Setor Borracha, que permitem comparar o relativo da periodização construída e dialogar com evidências acadêmicas.

Palavras-chave: Indústria. Negócio. Borracha. FUNSA. Uruguay.

\section{RESUMEN}

Este artículo busca, desde la Historia de Empresas y Empresarios, y su variedad de miradas, analizar el caso de la Fábrica Uruguaya de Neumáticos S.A. (FUNSA), procesadora de productos del caucho y goma en el periodo 1935-1974, que domina el mercado de Uruguay en ese periodo. Su fundación con capitales nacionales, desarrolo explosivo y diversificación productiva basada en la protección legislativa, el estancamiento y repunte, sirven de caso emblemático de la industria local representando en forma sintética el camino seguido por ella. A su vez se realizan variadas estimaciones de Valor Agregado Bruto, Ocupación y Salarios de la empresa y el Sector Caucho que permiten cotejar el relato de periodización construido así como dialogar con evidencias académicas.

Palabras-clave: Industria. Empresa. Caucho. FUNSA. Uruguay.

* Agradezco al profesor Raúl Jacob por sus sugerencias para este artículo.

Fecha de recepción: 13 de octubre de 2017; fecha de aceptación: 1ํ de julio de 2018.

$\star \star$ Profesor de historia y candidato a Magister en Historia Económica en la Facultad de Ciencias Sociales de la Universidad de la República, Uruguay. E-mail: ssabini@gmail.com 


\section{Introducción}

Este artículo ${ }^{11}$ aborda la historia económica de la empresa procesadora de productos del Caucho, Fábrica Uruguaya de Neumáticos Sociedad Anónima (FUNSA) en el período 1935-1974, la cual ha sido calificada como monopólica en el mercado uruguayo (Aguirre; Dellepiane; Duarte, 2004, p. 37; González Sierra, 1991, p. 3-4), aspecto que será discutido a lo largo del mismo.

La hipótesis de trabajo refiere a la posibilidad de representar la historia industrial del Uruguay través del Sector Caucho, a través de la empresa FUNSA, cotejando si responde al relato de la industria de Uruguay de un periodo de desarrollo explosivo y diversificación productiva, entre 1930-1954, una década de estancamiento, y la recuperación hacia comienzos de la década de 1970 (Bértola, 1991; Finch, 2014).

Se ha definido una serie de tópicos en función de las fuentes disponibles y para su análisis se han establecido dos periodos que tienen un punto de quiebre con los primeros problemas financieros que enfrenta la empresa.

El primer periodo "La empresa FUNSA apertura, auge y desarrollo (1935-1958)" considera el origen de capitales que fundaron la empresa; la estrategia expansiva que siguieron los empresarios y una estimación delVBP de la empresa y sector caucho; las dificultades de acceso a las materias primas; la expansión y diversificación productiva entre 19451958; las relaciones laborales en el período 1942-1958 y una estimación salarial comparada de FUNSA, Sector Caucho y Uruguay.

El segundo, denominado "FUNSA primeros tropiezos y cambios de estrategia (1958-1974)" aborda la estrategia empresarial considerando las estimación del IVF del Sector Caucho y su correlato con la empresa; la relación con Firestone, el financiamiento bancario; la estrategia lobbista; el relacionamiento con los trabajadores en 1958-1974 y la estrategia exportadora.

Por último se trazan las primeras conclusiones que intentan vincular algunas de las hipótesis plasmadas y su relación con el marco teórico de

Representa una avance de la Tesis de Maestría en Historia Económica y Social "Breve Historia de la Empresa FUNSA en el Uruguay (1935-2002)" bajo la tutoría del Mag. Juan Pablo Martí, en el marco del Programa de Historia Económica y Social de la Facultad de Ciencias Sociales de la Universidad de la República, Uruguay. 
referencia relativo a algunos postulados institucionalistas, chandlerianos y postchandlerianos.

\section{Fundamentación, justificación y antecedentes}

La Historia de Empresas y Empresarios en Uruguay en el contexto latinoamericano se encuentra en un bajísimo desarrollo, fundamentalmente por problemas de masa crítica (Jacob, 2008, p. 169-177). Sin embargo, es posible acercarnos al caso a través de diferentes investigaciones que lo integran o mencionan en forma indirecta.

Uruguay tiene un amplio debate sobre la evolución de su Producto Bruto Interno en el siglo XX y sobre el Producto Industrial, a partir del mismo es posible acercarse a estudios que integran el Sector Caucho, que sin ser el eje de estas investigaciones son trascendentes para este artículo (Millot; Silva; Silva, 1973; Finch, 2014; Jacob, 1981; Bértola, 1991; Arnabal; Bertino; Fleitas, 2013; Bértola; Bittencourt, 2014). En el mismo sentido son de relevancia, por sus referencias al mismo Sector, las publicaciones realizadas desde instituciones públicas con fines estadísticos y académicos (CGN, 1941; BROU, 1965; BCU, 1969; IECON, 1969; FCS, 2005).

Desde la economía existe la Tesis de Grado de Aguirre, Dellepiane y Duarte (2004) la cual realiza una breve reseña de los aspectos histórico-económicos. Diferentes estudios sobre FUNSA adoptan diferentes miradas, en su reapertura como organización autogestionada por sus trabajadores (Cabral; Chacharro; Misevicius, 2006). Desde la relación salarial en un marco comparativo (Chevalier; Fernández; Motta, 2006; Fernández Caetano, 2012), estudios históricos desde la mirada sindical (González Sierra, 1991, 1998), y de forma indirecta a través de las inversiones de Estados Unidos en Uruguay (Azar; Fleitas, 2010).

Desde la mirada de la Historia de Empresas y Empresarios investigar el caso de FUNSA, supone discutir el carácter de empresa monopólica de manufactura de productos de caucho y goma, desentrañar su derrotero así como una serie de especificidades del desarrollo de la industria del Uruguay desde la década mediados de 1930 hasta 1974. 


\section{Metodología}

El artículo trata de un estudio de caso, despliega análisis heurístico y hermenéutico de fuentes primarias y secundarias, y se nutre de diversas técnicas y estrategias propias de la historia empresarial, a través del método inductivo-deductivo. Supone el análisis de las fuentes del fondo de archivos de la empresa FUNSA, compuestos por más de 2.500 folios, al cual se tuvo acceso, así como prensa de la época. Asimismo busca establecer un relato de periodización de la empresa que considere las diferentes etapas que le tocó enfrentar a la luz de los cambios económicos e institucionales cotejando los mismos con diferentes indicadores.

\section{Marco teórico}

El marco teórico de referencia, opera como una guía por el cual se transita a través de elementos centrales, no como un marco rígido preestablecido. Asume que las instituciones afectan el desarrollo y desempeño empresarial (North, 1990) y a través de estrategias de desarrollo escala y especialización de sus gestores las empresas buscan posicionarse para dominar mercados (Chandler, 1990), y que ante los cambios institucionales las empresas desarrollan estrategias de adaptación y aprendizaje basadas en sus redes de apoyo políticas, familiares y empresariales (Zeitlin; Sabel, 1997).

\section{El producto industrial en Uruguay en el periodo 1930-1974}

Las miradas más recientes sobre la evolución de la industria en el Uruguay marcan un periodo sostenido desarrollo y crecimiento entre 1930-1954, una meseta hasta principios de la década de 1970 (Bértola; Bittencourt, 2014, p. 3). A través de indicadores comoVBP, Ocupación, Salarios e IVF se verá que en trazos generales la empresa FUNSA responde a este relato en sintonía con el Sector Caucho.

Nuestra empresa nace bajo el paradigma de la Industrialización por Sustitución de Importaciones (ISI), renombrado como Industrialización Dirigida por el Estado (IDE) 1930-1972 (Bértola; Bittencourt, 2014). 
la industria manufacturera se convierte en este periodo en el sector dinámico que luego de la crisis determinó la recuperación del producto bruto interno. En 1938 el producto industrial supera los niveles de 1930 en un $53.9 \%$ lo que muestra un crecimiento particularmente acelerado de este sector. (Millot; Silva; Silva, 1973, p. 93)

Las industrias procesadoras de materias primas nacionales, mayormente agrarias, fueron las protagonistas de este proceso. FUNSA jugaría un papel más lateral y al mismo tiempo particular, ya que se trataba de una de las pocas industrias que procesan materias primas importadas. En esta "Edad de Oro" del sector industrial, el peso de la industria pasó del 12.5\% del PBI en 1930 al 20.3\% en 1950 (Millot; Silva; Silva, 1973).

El caucho sería una de las ramas de mayor expansión, Bértola (1991, p. 206) ha estimado la variación de VAB, Ocupación, Productividad y Salario Real para el período 1943-1954. Allí encontramos con que para ese periodo la industria del caucho tuvo una variación de $31.6 \%$ de $\mathrm{VAB}, 11.5 \%$ de ocupación, $18 \%$ de productividad y $7.8 \%$ de salario real, por encima de las 14 ramas estudiadas, equipos de transporte la seguía en $\mathrm{VAB}$ con $21.2 \%$ y $14,7 \%$ en productividad, Metales en Ocupación con $9,1 \%$, y solo por debajo del tabaco en Salario Real con $8,9 \%$. Sin duda se trata de un desempeño extraordinario.

Este periodo expansivo de la industria entra, a partir de 1954, en una década de estancamiento provocada fundamentalmente por la caída de los términos de intercambio de los productos agropecuarios que eran la base del sistema de cambios múltiples y la importación de insumos para la industria y bienes de consumo (Bértola; Bittencourt, 2014, p. 65-66).

\section{La empresa FUNSA: apertura, auge y desarrollo (1935-1958)}

\subsection{La cuestión del origen del capital y su concentración}

Habilitada desde octubre de 1935 (González Sierra, 1991, p. 3) la empresa tiene unos primeros años que implican la reunión del capital accionario, la compra de equipamiento, contratación del personal, y el comienzo de las ventas. En setiembre de 1936, diría su presidente en la primera reunión de accionistas: "Este primer ejercicio ha sido dedicado 
a la integración del capital y construcción de la fábrica, en consecuencia no han habido operaciones desde que las ventas recién se ha iniciado con posterioridad al cierre del ejercicio" (FUNSA, Actas Asambleas de Accionistas, libro 1, 1936, p. 5).

El proceso productivo de FUNSA fue definido como taylorista, obreros ejecutores con tareas simples y rutinarias, métodos y procesos de trabajo estandarizados (González Sierra, 1991, p. 5-6) y fuerte supervisión al trabajo obrero (Aguirre; Dellepiane; Duarte, 2004, p. 26).

Se ha afirmado por Azar y Fleitas, siguiendo la tradición historiográfica (Oddone, 1989; Oficialdegui, 1995; Jacob 2000; Finch, 2014), que la empresa fue fundada con capitales norteamericanos:

El primer episodio posterior a 1930 que puede verificarse en la literatura transcurre en 1936, cuando arriban a Uruguay inversiones directas destinadas a la producción de manufacturas, destacándose la instalación de la Fábrica Uruguaya de Neumáticos S.A, FUNSA, por parte de la Goodrich Tyre Company. (Azar; Fleitas, 2010, p. 15)

Jacob indica que se trataba de una confusión, advertida por la empresa en la época, aclarada a través de un comunicado en el diario La Mañana, del 4 de diciembre de 1938, indicando "que el capital invertido era local y que pertenecía a casi quinientos accionistas" (Jacob, 2016, p. 154). En ese sentido, la empresa realizaría una serie de publicaciones en ocasiones conmemorativas, hacia 1940 en el diario El Pueblo indicaba que FUNSA tenía de capitales totalmente uruguayos (El Pueblo, 25 de agosto de 1940) y en 1943 en el diario El País:

Bajo dirección uruguaya, con capitales uruguayos, con personal criollo en su inmensa mayoría, y con directrices técnicas de Goodrich, la gran fábrica de neumáticos norteamericana, la F.U.N.S.A., ha recorrido en forma sistemática y denodada, en 7 breves años, el largo, lento y azaroso camino, que la mayoría de las industrias sudamericanas ha necesitado para imponerse en los mercados. (FUNSA, El País, 11 de julio de 1943, p. 9)

Surge tanto en las Actas de Accionistas, como en las reuniones de Directorio, la prensa local, y la obra de Jacob (2016) que la relación con las empresas de Estados Unidos refiere a una consistente y permanente asistencia técnica, como una línea de larga duración. Como se indicó, 
la dirección técnica de la instalación de la planta se realiza por dos ingenieros representantes de BF Goodrich (FUNSA, Actas Asambleas de Accionistas, libro 1, Acta n. 3, julio de 1937, p. 18).

En la primera reunión de accionistas realizada en setiembre de 1936, a la cual concurren sesenta y seis de ellos, representado más del 50\% del capital accionario (3.569 acciones), surgen nombres criollos y de origen nacional, no aparecen apoderados de Goodrich Tyre Company. Destacan sí algunos de los que a la postre serían los directivos determinantes de la empresa por varias décadas: Pedro Sáenz, Diego Arocena Capurro y Saturnino Fernández (FUNSA, Actas Asambleas de Accionistas, libro 1, setiembre de 1936, p. 1-4).

La concentración del capital accionario en algunos pocos empresarios es un proceso que se puede identificar en los primeros veinte años de la empresa. Estos empresarios serán los mismos que llevan adelante el Directorio de la empresa, dotando a los mismos de una concentración de poder muy grande. Actuando al mismo tiempo como gestores y propietarios. El mecanismo era más bien sencillo, los dividendos de la empresa podían cobrarse o bien en acciones o bien en capital, sistemáticamente algunos directivos, cobran sus dividendos en acciones, ello les permite, no solo tener una mayor retribución futura, sino establecer una alianza política determinante en la elección del Directorio (FUNSA, Actas Asambleas de Accionistas, libro 1, Acta n. 2, setiembre de 1937, p. 15).

Ese mismo año se propone un cambio profundo en los estatutos, el cual dotará de un poder muy especial al Directorio, con el argumento de no contraponer intereses de accionistas y directorio, se propone que el presidente cobre el $2 \%$ de utilidades y $1 \%$ por cada director, a su vez se crea un fondo de reserva de $5 \%$ y el $95 \%$ finalmente se cobra por los accionistas. Al mismo tiempo, se amplía el mandato a cuatro años y la renovación de la mitad del directorio cada dos años (FUNSA, Actas Asambleas de Accionistas, libro 1, Acta n. 3, julio de 1937, p. 21-31).

De esta forma mientras que en 1936 Pedro Sáenz (presidente) y Diego Arocena Capurro (secretario), tenían 600 y 279 acciones sobre 3.569 presentes, es decir 24.6\%, una década después, en 1945 ya acumulaban un total de 9.421 y 1.083 respectivamente sobre 24.294 acciones presentes, lo que representa un 43.2\% (FUNSA, Actas Asambleas de Accionistas, libro 1, Acta n. 13, setiembre de 1945, p. 153). 


\subsection{La estrategia expansiva}

El capital accionario es un tema permanente en las asambleas anuales, se identifica un proceso de crecimiento del mismo durante un periodo sumamente prolongado. Está claro que este capital accionario iba en aumento en la medida que la empresa se expandía multiplicando por varias veces su capital accionario entre 1936 y 1945 (FUNSA, Actas Asambleas de Accionistas, libro 1, Acta n. 3, julio de 1937; n. 13, setiembre de 1945). La estrategia expansiva se basa en la captación de ahorro privado con un mercado local cautivo, identificando un proceso de diversificación productiva y compra de empresas ya instaladas, este relato expansivo parece ajustarse al que se produjo en la industria uruguaya de la época.

En 1936 se compra una fábrica de artículos de goma en Montevideo, Buscanello y Lind, sus activos y pasivos por 150 mil pesos (120 mil dólares), a través de la emisión de acciones por ese monto (FUNSA, Actas Asambleas de Accionistas, libro 1, Acta n. 2, setiembre de 1936). Tempranamente, ya en 1938, se plantea una ampliación de las actividades de la empresa con la fabricación, además de cubiertas y cámaras, de todo tipo de productos de goma: bolsas, caños, pelotas, telas engomadas, ligas, tacones, calzados (FUNSA, Actas Asambleas de Accionistas, libro 1, Acta n. 5, agosto de 1938).

Gráfico 1 -Valor bruto de producción de FUNSA y sector caucho de Uruguay, 1937-1946 (miles de pesos constantes de 1936)

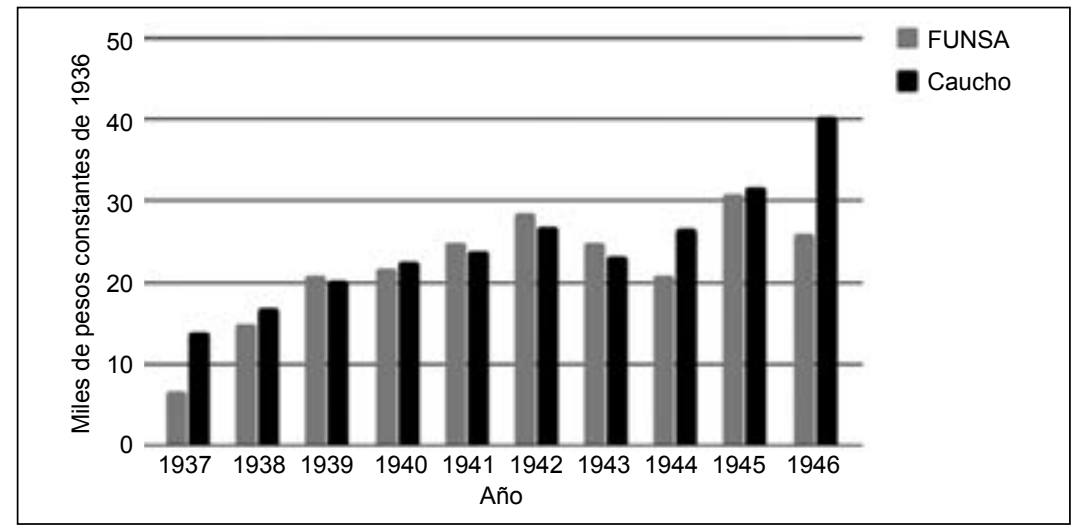

Fuente: elaboración propia en base a FUNSA, Memoria y balance (1937-1946); para Sector Caucho: 1936-1939 en CGN - Contaduría General de la Nación (1941, p. 267); 1940-1941 en Millot, Silva y Silva (1973, Cuadro 5); 1942-1946 en Bértola (1991, p. 287). 
Para diversificar la producción, en 1938, se decide la compra de INCAL, empresa dedicada a la producción de calzado y productos de goma, con éxito evidente en 1942 ya había aumentado un 38\% sus ventas (FUNSA, Actas Asambleas de Accionistas, libro 1, setiembre de 1942, p. 90). En la misma línea, en setiembre de 1944, se compra Fibra Vegetal S.A. para el aprovisionamiento de materia prima para algunos de los productos, y también se hicieron inversiones en Compañía Caucho Aglomerado S.A. con el objetivo de complementar la línea de calzado (FUNSA, Actas Asambleas de Accionistas, libro 1, setiembre de 1942, p. 120).

El Gráfico 1, muestra por un lado la tendencia creciente de la valor de la producción de FUNSA, lo cual confirma el éxito de la estrategia seguida, y que en estos primeros años FUNSA “empuja” en forma creciente al Sector Cacho, por otro lado, y en este sentido que para la década 1937-1946 la totalidad de la producción y ventas de las manufacturas del caucho se corresponden con FUNSA, por lo que la idea de monopolio parece ajustada.

\subsection{Las dificultades del acceso a las materias primas en los primeros años}

La empresa FUNSA producía cubiertas con materias primas importadas, un hecho relativamente particular para la industria de Uruguay, negro de humo y caucho eran las principales para la producción. La Segunda Guerra Mundial la dejaría en una situación grave, si la dependencia de materias primas ya era un gran desafio en tiempos de paz, la guerra afectaría tanto por el acceso a los mercados de India, Inglaterra y Holanda como por la propia necesidad de caucho de Estados Unidos, para resolver este problema la estrategia seguida por FUNSA sería de acopio de las materias primas (FUNSA, Actas Asambleas de Accionistas, libro 1, julio de 1940, p. 85).

Las trabas tenían que ver con demoras en los permisos de importación, certificados de prioridad, e incluso la escasez por el hundimiento de barcos (FUNSA, Actas Asambleas de Accionistas, libro 1, setiembre de 1942, p. 90-91). El punto máximo de esta situación fue la expropiación por parte del gobierno de Estados Unidos de 470 toneladas de caucho propiedad de FUNSA, ofrecía a pagar el equivalente del valor en caucho proveniente de Brasil más las ganancias. Empresa y gobierno uruguayo 
trabajaban juntas y esperaban que Estados Unidos establezca una cuota de exportación de caucho para las necesidades del país (FUNSA, Actas Asambleas de Accionistas, libro 1, 1942, p. 90-101).

\subsection{Expansión y diversificación productiva (1945-1958)}

La lista única de Pedro Sáenz y Diego Arocena Capurro continúa dominando la empresa entre 1945 y 1958. Entre 1945 y 1946 se presenta un plan para la expansión de la empresa, la primera etapa supone la construcción de un edificio para produccion de baterias de autos, ómnibus, camiones y también baterías estacionarias para granjas y estancias. Otros edificios nuevos, para procesar materias primas, para la filial INCAL, así como para la denominada "obra social" de la empresa que suponía salas médica y dental, comedores, sala de lectura y descanso para empleados y obreros, y cooperativas de consumo. A ello sumado la compra de de 51.590 metros cuadrados a terrenos con salidas a tres calles con el objetivo de ampliar las instalaciones (FUNSA, Actas Asambleas de Accionistas, libro 1, Acta n. 13, setiembre de 1945).

Asimismo se incorporan nuevas máquinas para procesar caucho sintético y nuevas telas, con el objetivo de mejorar los productos, los cuales requirieron adiestramiento del personal técnico y de producción "realizando ensayos dificiles y costosos". Se busca el perfeccionamiento de la producción y la calidad comienza a ser una preocupación, estableciendo como parámetro que "hay un número de pocos reclamos en relación al número de ventas" (FUNSA, Actas Asambleas de Accionistas, libro 2, Acta n. 16, junio de 1948).

Este proceso expansivo se ve impulsado por la demanda interna, que no puede ser satisfecha. Los directivos atribuyen a la organización del trabajo y a nivel técnico una "triplicación de la producción en los principales renglones", a modo de ejemplo, mientras antes de la guerra se producían 6.000 cubiertas Heavy Duty en 1946 se producen $30.000 \mathrm{al}$ año, cinco veces más (FUNSA, Actas Asambleas de Accionistas, libro 1, Acta n. 14, octubre de 1946).

Hay que destacar que en este período, todo el plan de expansión, que suponía tanto la construcción de nuevos edificios, como la compra de empresas se llevaba adelante con la emisión de acciones, lo cual indica una captación del ahorro nacional, lo mismo que con los dividen- 
dos generados por la propia producción y que los accionistas, en lugar de cobrar en efectivo, lo hacían en acciones, posibilitando tales inversiones, a modo de ejemplo, en 1946 ante la necesidad de capitales erase emitieron hasta 10.000 acciones "en lugar de tomar crédito bancario" (FUNSA, Actas Asambleas de Accionistas, libro 1, Acta n. 14, octubre de 1946).

El Plan supone también la adquisición de plantas ya instaladas, no únicamente para ampliar las capacidades productivas, como en el caso de la Fábrica Uruguaya de Conductores Eléctricos S.A. que produce cables eléctricos revestidos en caucho, sino también para obtener la porción de mercado de la competencia. Ejemplo de ello representa la compra de Caucho Aglomerado S.A que producía calzado económico con suela de caucho, la cual sería liquidada al año siguiente de su adquisición (FUNSA, Actas Asambleas de Accionistas, libro 1, Acta n. 14, octubre de 1946).

Luego del éxito del primer plan de inversiones y ampliaciones, en el quinquenio 1953-1957, se propone un nuevo proceso expansivo. La estrategia a seguir, además de continuar ampliando la planta física, tendrá que ver con la incorporación de asesoramiento técnico proveniente de Estados Unidos y Europa para la mejora de procesos e incorporación de tecnología y maquinaria diversificando la producción. Para la línea principal, abandonando a Goodrich Tyre Company, se cuenta con el asesoramiento de United States Rubber en el periodo 1945-1960 (FUNSA, Actas Asambleas de Accionistas, libro 1, octubre de 1946, s.p.), una de las empresas de mayor trayectoria en la fabricación de neumáticos de caucho sintético, para mejorar el ordenamiento de la mercadería, así como su envasado mejorando aspectos como la economía, protección y cuidado de los artículos, orden y control (FUNSA, Actas Asambleas de Accionistas, libro 2, Acta n. 27, setiembre de 1957).

Se contrató la asesoría de Technik and Handels AFA destacada por FUNSA como la "principal fábrica de batería y pilas secas de Europa" y la General Shoe Corp una de las más importantes fábricas de calzado de EEUU (FUNSA, Actas Asambleas de Accionistas, libro 2, Acta n. 22, setiembre de 1953), llegando en 1955 la nueva maquinaria. La producción apunta al mercado interno y al crecimiento vegetativo del país (FUNSA, Actas Asambleas de Accionistas, libro 2, Acta n. 25, setiembre de 1955). 
En el año 1954 comenzaría la fabricación de ruedas de bicicleta, bajo la técnica de Pirelli, y en 1956 se continuaría adelante con tubos de bicicleta y cubiertas, con la misma empresa. También se comienzan a producir pilas y baterías (FUNSA, Actas Asambleas de Accionistas, libro 2, Acta n. 26, setiembre de 1956).

Gráfico 2 - Establecimientos, ocupación de obreros y empleados en el sector caucho y FUNSA, 1930-1963*

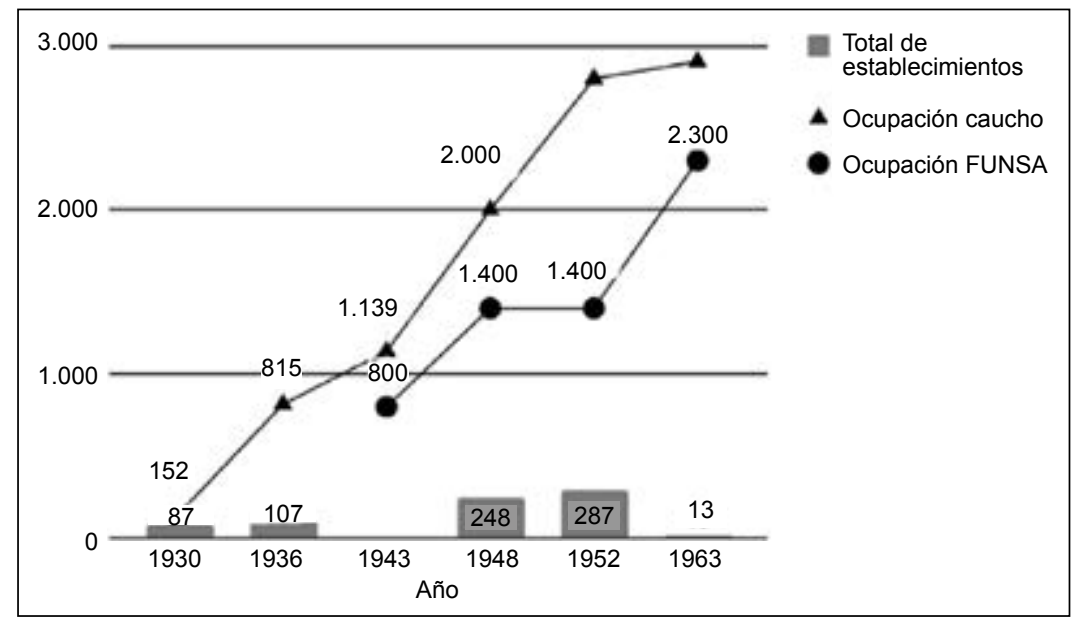

Fuente: elaboración propia establecimientos y ocupación del Sector Caucho en base a FCS (2005), IECON (1969). Datos FUNSA en base a 1940 (El Pueblo, 25 de agosto de 1940), 1946 (El País, 16 de febrero de 1946), 1952 (FUNSA, Actas Asambleas de Accionistas, libro 2, Acta n. 22), 1963 (FUNSA, Actas Asambleas de Accionistas, libro 2, Acta n. 27, n. 35).

* A los efectos de tener un cuadro comparativo, siendo que no existen datos homogéneos para el Sector Caucho y FUNSA, se realizan los siguientes aclaraciones: no hay datos para establecimientos de 1943; tampoco se cuentan con datos de la ocupación de FUNSA al comenzar sus actividades hacia 1936, para 1948 se toma el dato de 1946.

Dentro de los planes de la empresa en 1956 se busca ampliar la producción de motores eléctricos, transformadores, ventiladores domésticos, industriales y comerciales, y centrifugadoras con el asesoramiento de una empresa europea, y también la expansión de la producción de productos de látex (FUNSA, Actas Asambleas de Accionistas, libro 2, Acta n. 26, setiembre de 1956).

Como vimos antes este proceso se asienta sobre la expansión del capital accionario habilitado, dado que la empresa cotiza en la Bolsa de Comercio desde 1941 (Millot; Silva; Silva, 1973), captando en forma 
constante el ahorro interno. Hay que indicar que este proceso expansivo supone no solo el aumento de las ventas, diversidad de productos, y como veremos luego, también del porcentaje de captación sobre las ventas totales.

La expansión queda marcada en el proceso de concentración de la ocupación del sector en tanto en número de trabajadores como en la disminución del número de establecimiento de caucho (Gráfico 2). Se puede afirmar que el periodo 1952-1963 supuso un afianzamiento de las condiciones monopólicas que la empresa detentaba en el sector caucho en línea con los autores que hemos citado (Aguirre; Dellepiane; Duarte, 2004, p. 37; González Sierra, 1991, p. 3-4).

\subsection{Las relaciones laborales en el período 1942-1958}

Si bien no se tiene un dato exacto de la evolución del número de trabajadores, según las actas de la empresa y publicaciones hechas en prensa, FUNSA contaba con 800 trabajadores (obreros y empleados) hacia 1940-1943, en 1946 se había expandido hasta 1400 y en 1957 ese número había trepado a 2500 , dato de mayor ocupación registrado. A su vez la empresa cada vez más concentra la ocupación del sector (Gráfico 2).

La primera noticia que tenemos de un conflicto data de agosto 1942, desatado por la expulsión del secretario general del Sindicato de la Goma, Mario Gregores, y los dirigentes Celeste Pérez, Font y Piriz, desatando una huelga. Para su levantamiento tuvo que intervenir el presidente de la República, lo que marca la enorme repercusión que causó (González Sierra, 1991, p. 12). Las huelgas y ocupaciones serían una constante en el período, en 1946 se producen 96 días de huelga, que según la empresa provoca una caída de 6.69\% en ingresos (González Sierra, 1991, p. 16).

Si bien los Consejos de Salarios ${ }^{2}$ producían ciertas molestias en la empresa la evaluación es de cierta utilidad, hacia 1949 los directivos expresan:

2 Los Consejos de Salarios suponen la negociación tripartita de la remuneración que reciben los trabajadores; para una explicación de su surgimiento en Uruguay, ver Sarthou (2004); para un análisis comparativo sobre sus resultados, se puede ver Fernández Caetano (2012). 
Las relaciones de la empresa, con su personal, una de las preocupaciones más graves comunes a toda la industria y al comercio, debido al desorden que ha creado el funcionamiento defectuoso de los Consejos de Salarios son satisfactorias. - En breve se celebrará un nuevo convenio colectivo que, si bien causará cuantiosas erogaciones, presumiblemente permitirá trabajar con tranquilidad hasta fines de 1950. (FUNSA, Actas Asambleas de Accionistas, libro 2, Acta n. 16, octubre de 1949, s.p.)

Tras unos años de relativa calma, la empresa con un récord de ventas, en 1952 se firma un nuevo Convenio Colectivo, Carlos Sapelli, síndico de la empresa expresa:

"FUNSA tiene un Convenio Colectivo de sueldos y jornales, por un año de vigencia, pero por parte del personal, contagiado con el ambiente general de desorden que ha reinado en el país, intentó desconocer el convenio y se tuvo que tomar algunas medidas disciplinarias, pero que hoy está más tranquilo".Y el Presidente Pedro Sáenz: “[...] vamos saliendo de este estado un poco desordenado desde hace algunos días, debido a la acción enérgica y patriótica de los hombres de gobierno". (FUNSA, Actas Asambleas de Accionistas, libro 2, Acta n. 21, setiembre de 1952, s.p.)

Las relaciones entre gobierno y empresa parecen fructíferas en esta época, en contrapartida durante 1952-1957 un conflicto prolongado sería lo natural en la relaciones entre la empresa y el sindicato. José Claudio Williman (h), en forma premonitoria escribía hacia 1953:

La periodicidad de las huelgas de FUNSA ya comienza a adquirir estabilidad. Se debe ello a la situación por la que pasan Sindicato y Empresa; aquel, afirmando su autonomía y su eficacia como órgano de defensa de los derechos obreros; ésta, pendiendo paulatinamente su ascendente sobre los dirigentes gremiales, sin que aún se haya conformado a su nueva situación. (Williman (h), 1953)

Es que a fines de 1952 tomaba forma la fundación de la Unión de Obreros, Empleados y Supervisores de FUNSA (el sindicato), quien cumpliria un rol central en las relaciones laborales con la empresa y en el movimiento sindical del Uruguay (El Día, 11 de noviembre de 1962).

El conflicto se disparó por el despido de los dirigentes del sindicato (El Sol, 4 de noviembre de 1952). Medios socialistas denunciaban bajos 
salarios, la intención de formar un sindicato sumiso y amenazas por parte de la empresa (El Sol, 4 de noviembre de 1952, p. 2) y uno de sus legisladores, el Dr. Arturo J. Dubra, presionaba en el parlamento (El Sol, 9 de diciembre de 1952, p. 2), a fines de 1952 parecía que los trabajadores serían retomados, luego que la planta estuviera ocupada del 12 al 22 de diciembre y según el compromiso ante la Comisión de Legislación del Trabajo de la Cámara de Representantes ( $E l$ Sol, 30 de diciembre de 1952, p. 2).

En enero de 1953 los trabajadores eran finalmente retomados, el sindicato cargaría contra el ministro de Industrias y Trabajo, como el responsable, "el Dr. Grauert ha sido uno de los principales causantes de nuestro conflicto" (El Sol, 20 de enero de 1953, p. 2).

Durante 1954 se sucedían conflictos similares por despidos, paros y reposición de los trabajadores cesados (El Sol, 20 de enero de 1954, p. 4; 9 de julio de 1954), parte del conflicto entre el recientemente creado sindicato y el Directorio de la empresa, además, ese año debían convocarse nuevamente los Consejos de Salarios para lo cual junto con otros gremios se conformaba la movilización (Caggiani, El Sol, 4 de agosto de 1954), el salario tendría un aumento de 11\% según la empresa (FUNSA, Actas Asambleas de Accionistas, libro 2, Acta n. 23, setiembre de 1953), la tendencia creciente coincide con a la estimación realizada (Gráfico 3).

En 1955 se produce una nueva huelga, esta vez de de 53 días, denunciando las condiciones de trabajo en FUNSA, y como ya hemos visto con el apoyo de los legisladores socialistas, en este caso Germán D'Elía denunciaba Lock-out patronal y que la empresa se aprovechaba de las condiciones de protección que le daba el Estado y explotaba a los trabajadores en un régimen militar (El Sol, 24 de abril de 1955; 11 de mayo de 1955, p. 2; junio de 1955, p. 2).

En 1956, los conflictos se sucedían en forma casi idéntica (El Sol, 12 de octubre de 1956; 26 de octubre de 1956; 23 de noviembre de 1956), resulta significativo que en las actas de las asambleas de accionistas no se mencionan los conflictos, más preocupados por la cuestión impositiva (FUNSA, Actas Asambleas de Accionistas, libro 2, Acta n. 26, setiembre de 1956). Sin embargo, la empresa publicaba en la prensa, tanto en notas firmadas por la Gerencia (El Debate, 15 de diciembre de 1956, p. 2) como por el presidente (El Debate, 19 de diciembre de 1956), sus razones 
respondiendo al sindicato cada uno de sus argumentos, aún luego de haber firmado el convenio el 26 de noviembre de ese año.

Gráfico 3- Salario real comparado FUNSA, industrias del caucho y salario mensual de Uruguay (pesos constantes de 1936)

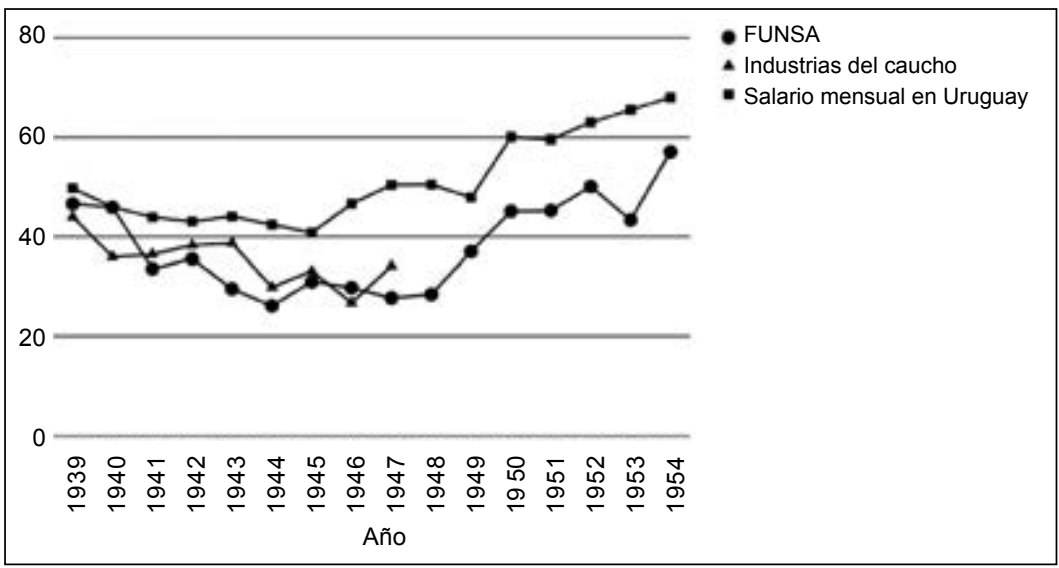

Fuente: elaboración propia en base a FUNSA, Libros de egresos e ingresos (1936-1958); Industrias del Caucho en Millot, Silva y Silva (1973); Salario Mensual de Uruguay en Bértola et al. (1999).

FUNSA continuaría con una práctica permanente, la de publicitar por medio de la prensa sus conflictos, como hemos visto no sólo con los trabajadores sino con otras empresas, en esta oportunidad, indicaba que los trabajadores rechazan una propuesta que les otorgaba los salarios más altos de la industria (Acción, 12 de julio de 1957, p. 9).

La estimación salarial (Gráfico 3) muestra que por un lado los salarios de FUNSA se encontraban por debajo de los salarios del sector (aún cuando esta era la principal empresa) y por debajo del promedio del Salario Nominal promedio del país. Al mismo tiempo que acompañaba los movimientos de ascenso y caída de ambos.

Los Consejos de Salarios, instalados desde 1943, parecen haber operado en forma positiva para la recuperación y aumento salarial, tanto en FUNSA como en la mejora general de los salarios en Uruguay entre 1943 y 1955. 


\section{FUNSA: primeros tropiezos y cambios de estrategia (1958-1974)}

\subsection{La estrategia empresarial en un periodo de dificultades}

Si a fines de los 50 la estrategia de la empresa para seguir desarrollando inversiones era la de pagar dividendos bajos, se pasaría directamente a no pagarlos por razones financieras y luego a solicitar financiación bancaria nacional e internacional. En apenas quince años, la situación económica nacional obligó a la empresa a cambiar su tradicional forma de hacer las cosas, destacan de este periodo la apertura de los mercados de Argentina y Brasil, que al final del mismo, llegaron a niveles sumamente relevantes.

Las perspectivas en 1960 eran malas:"El balance de la sociedad refleja las desalentadoras perspectivas en informes de anteriores ejercicios. La inflación ha golpeado con intensidad creciente nuestros rubros básicos" (FUNSA, Actas Asambleas de Accionistas, libro 2, setiembre de 1960). Los diez años siguientes estarían marcados por la alta inflación, tema mencionado en todas las asambleas de accionistas, la cual provoca caída de ventas y aumento del costo de vida, ese año se perderían 167 puestos de trabajo.

En 1962, el presidente Saturnino Fernández, habiendo fallecido en histórico presidente Pedro Sáenz, expresaba:"No ha sido nada agradable para nosotros el poder presentar a Uds., Sres. Accionistas, el resultado de este ejercicio. [...] No es posible pronosticar en qué forma terminará la crisis que afecta a toda la industria nacional, agobiada por los aumentos de costos y la falta de liquidez y disponibilidades".

Por primera vez no se pagan dividendos en efectivo para no cortar con proyectos productivos que tiene el directorio (FUNSA, Actas Asambleas de Accionistas, libro 2, Acta n. 35, octubre de 1962, p. 180).

Muestra de esta crisis se manifiesta por la gran capacidad ociosa existente en el sector caucho (proceso acompañado en toda la industria local), el Instituto de Economía de la Universidad de la República, estimaba que hacia 1963 en relación a la ocupación era de $29.1 \%$ y en función de la producción máxima 38.2\% (IECON, 1969, p. 58). En 1965 la empresa ya no era capaz de financiar las inversiones con recursos propios, lo que había sido una constante hasta ese momento (FUNSA, Actas Directorio, Acta n. 1.157, mayo de 1965, p. 548). 
Gráfico 4 - Índice de volumen físico, productos del caucho, Uruguay, 1955-1967

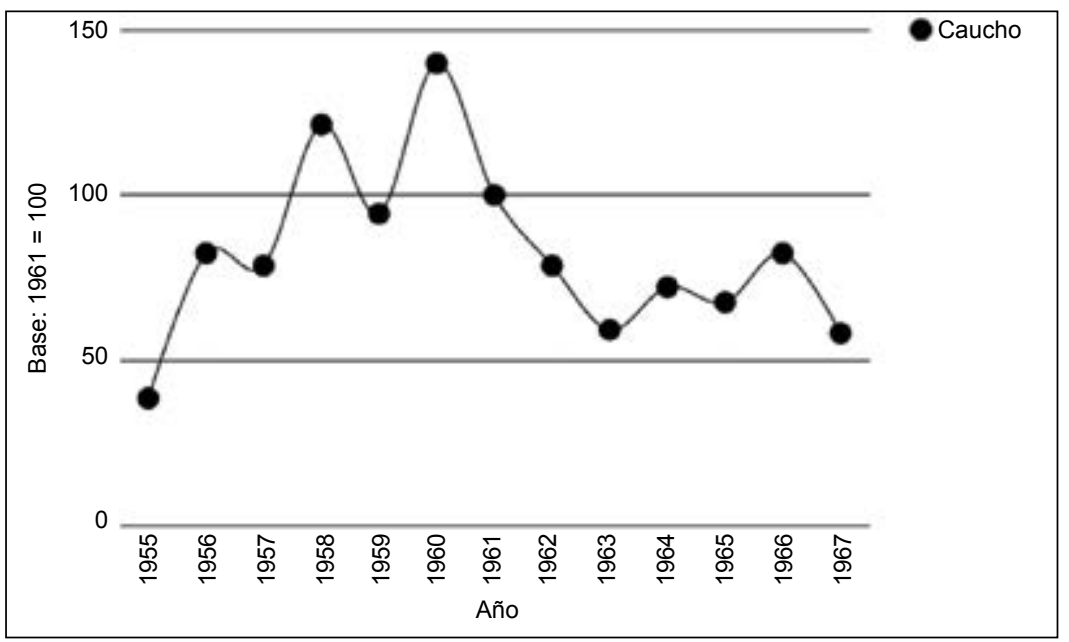

Fuente: elaboración propia en base a BCU (1969, Tabla 2.B.11).

Si analizamos la evolución del Índice de Volumen Físico de los productos del caucho en el periodo se ve con claridad el peso relativo de la empresa FUNSA en el sector, que parece determinante para su evolución, a la vez que confirma una década de 1950 pujante y una caída hacia inicios de 1960 que se mantiene en niveles relativamente bajos de producción, como veremos, la recuperación recién se daría con el impulso exportador. Nuevamente la evolución también coincide con la economía nacional que transitaba por un periodo de estancamiento, con la industria como uno de sus principales sectores (Bértola; Bittencourt, 2014).

Más allá de las dificultades, FUNSA apuesta a la compra de FANAESA dedicada a la fabricación de baterías, con una inversión de 60 mil dólares, "una fábrica bien instalada, amplia y muy bien presentada", su transformación llevaría algunos años, en 1968 se reciben los primeros balances y en 1969 serían positivos, aunque hacia fines de nuestro periodo, 1974, sufriría con la falta de materias primas. La empresa, si bien se manejaba con independencia, tendría como presidente a Tydeo Larre Borges y al Cr. A. Mujica, ambos accionistas de FUNSA (FUNSA, Actas Directorio, Acta n. 1.167, julio de 1965, s.p.; n. 1.316, setiembre de 1968, s.p.; n. 1.334, marzo de 1969, s.p.; n. 1.492, agosto de 1972, s.p.; n. 1.497, octubre de 1972, s.p.; n. 1.556, marzo de 1974, s.p.). 
También se amplían las instalaciones de poliuretano y remodelan edificios, tomando deuda prendaria del Banco de la República (BROU) por 57 mil dólares (FUNSA, Actas Directorio, Acta n. 1.196, marzo de 1966, s.p.), ampliando luego dicho préstamo prendario a 123 mil dólares (FUNSA, Actas Directorio, marzo de 1967, p. 689).

\subsection{La relación con Firestone}

Paralelamente sería un periodo de implementación del Convenio de Asistencia Técnica con Firestone, que ahora sustituye a U.S. Rubber, desde 1961 se valora cómo el acuerdo impacta en la calidad y economía de productos y desde esa época la empresa tiene prioridad en la compra de acciones de la propia FUNSA (FUNSA, Actas Asambleas de Accionistas, libro 2, Acta n. 33, setiembre de 1961, p. 156).

Este hecho fue valorado por González Sierra (1991, p. 153) como la "venta" de FUNSA a Firestone, esto no parece ser así ya que si bien tenía un representante en el Directorio y paquete accionario, no era quien tomaba las decisiones en la empresa, sino los accionistas históricos.

Aguirre, Dellepiane y Duarte indican que, con la llegada de Firestone, "se implementan importantes cambios, que apuntan a una mayor capacidad de la planta, accediendo a una tecnología más moderna, logrando una mejora sustancial en la calidad el producto. En estos años se efectuaron importantes inversiones en maquinarias, moldes y edificaciones" (Aguirre; Dellepiane; Duarte, 2004, p. 70).

De hecho la empresa valora en forma positiva el desempeño del acuerdo adjudicando a las mejoras en el balance como "parte del Convenio de Asistencia Técnica suscrita con 'Firestone', cuyas características y ventajas principales explica, los excelentes resultados que ya se notan en la calidad y economía de los artículos que se producen en la fábrica" (FUNSA, Actas Asambleas de Accionistas, libro 2, Acta n. 33, setiembre de 1961, p. 156).

Sin embargo resulta trascendente que cuando su representante se retira a Venezuela la empresa no solicitará sustituto (FUNSA, Actas Directorio, Acta n. 1.442, 1971), de hecho pocas noticias se tendrán de él, apenas un informe sobre nuevos neumáticos en 1969 (FUNSA, Actas Directorio, enero de 1969, s.p.).

Entre 1970 y 1971 se negocia las condiciones del contrato (FUNSA, 
Actas Directorio, Acta n. 1.402, julio de 1970; n. 1.443, junio de 1971; n. 1.451 , setiembre de 1971; n. 1.462, diciembre de 1971; n. 1.463, diciembre de 1971), la novedad del acuerdo alcanzado, aprobado por el Banco Central del Uruguay (BCU), además del asesoramiento que ya se venía produciendo, radica en que FUNSA se obligaba a producir cámaras, cubiertas y otros productos con destino a exportación y que se identifican como Firestone (FUNSA, Actas Directorio, Acta n. 1.469, enero de 1972; n. 1.503, octubre de 1972).

\subsection{El financiamiento bancario}

Hasta 1958 la mayor parte de la operaciones financieras e inversiones de la empresa se llevaban adelante con recursos propios, lentamente se iría desarrollando un lento pero continuo endeudamiento, fundamentalmente con el sector público. En forma creciente, el BROU financia compra de maquinaria, en conjunto con el Banco Interamericano de Desarrollo (BID), y el BCU realiza adelantos para importaciones.

En estos años el problema del abastecimiento de las materias primas sería de primer orden, fundamentalmente por la falta de divisas, agravada por la devaluación y los controles que realiza el BROU por las propias necesidades del gobierno. Incluso los bancos privados tenían dificultades para financiar las importaciones de materias primas afectados por el tipo de cambio (FUNSA, Actas Directorio, Acta n. 1.158, junio de 1965, p. 550). Lo cual llevaría incluso a replanificar la producción y hasta la detención de la misma en setiembre de 1965, ya que el propio ministro de Industria de gobierno informaba que iban a faltar materias primas (FUNSA, Actas Directorio, Acta n. 1.163, junio de 1965).

Esta situación, que se sumaba a la falta de financiamiento para inversiones, llevaría a una toma de medidas. Se plantea reducir gastos salariales, plan de egresos voluntarios y como ya vimos anular todo tipo de dividendos complementarios, ajustar descuentos y créditos de ventas (de $200 \%$ a 100\%), y por último la solicitud de préstamos bancarios y prendas al BROU por 875 mil dólares en 1965 (FUNSA, Actas Directorio, Acta n. 1.164, julio de 1965, p. 557).

Hacia 1968, uno de los problemas que enfrentaba la empresa era la prefinanciación de las importaciones, para ello se realizaron gestiones ante el BROU y los bancos privados, Mercantil, Italiano City y Financiero 
(FUNSA, Actas Directorio, Acta n. 1.324, noviembre de 1968). Ese año comenzaron las gestiones ante el BID para un préstamo de ampliación de la planta de neumáticos, proyecto que iría tomando forma y avanzando y en 1970 supondría 6 millones de dólares (FUNSA, Actas Directorio, Acta n. 1.297, mayo de 1968; n. 1.378, n. 1.381, enero de 1970).

Hacia 1972 Ingeniero Torres expresa en este sentido:"Se puso especial énfasis en la necesidad de mejorar la máquina de solucionado de telas y la tubera de extrudar bandas de rodamiento. Esta última máquina en particular se considera incapaz de entregar un producto acorde a las modernas necesidades técnicas" (FUNSA, Actas Directorio, Acta n. 1.468, enero de 1972).

Los préstamos también surgen de la banca pública, en 1970 se el BROU aprueba un préstamo por más de 100 mil dólares para maquinaria $^{3}$ en condiciones favorables, y en el mismo sentido se levanta un embargo que existía desde 1955 (FUNSA, Actas Directorio, Acta n. 1.461, noviembre de 1971). En 1972 se reitera un préstamo de 6 millones de dólares para operar con el descuento de conformes por la misma institución y el préstamo prendario sube a 9.2 millones de dólares, este con destino a la compra de maquinaria, el mismo se aumentaría nuevamente hasta 17 millones de dólares en 1972 y otros 1,6 millones en 1973 (FUNSA, Actas Directorio, Acta n. 1.480, abril de 1972; n. 1.474, marzo de 1972; n. 1.502, octubre de 1972; n. 1.522, mayo de 1973).

El BCU, financia las ventas a la empresa estatal de electricidad basándose en el Artículo 24 de la Ley n. 13.608, de 1964 (FUNSA, Actas Directorio, Acta n. 1.391, mayo de 1970), y en 1972 fija una cuota de importación de 5.6 millones de dólares para materias primas, equipos, repuestos, productos terminados (FUNSA, Actas Directorio, Acta n. 1.467, enero de 1972). Finalmente, ante el escaso préstamo otorgado finalmente por el BID (en función de las expectativas), sería el propio BROU que financia el plan de "Promoción del Desarrollo, ampliación y modernización de la planta de neumáticos” por 3.7 millones de dólares (FUNSA, Memoria y balance, octubre de 1972).

\footnotetext{
3 Para la fabricacion de neumaticos: 2 Bag O Matic (pasajeros y HS), balanceadora, 4 tambores para armar, equipo separador de negro de humo (un producto necesario para la fabricación de neumáticos), 2 zorras elevadoras, 8 moldes para cubiertas (FUNSA, Actas Directorio, Acta n. 1.402, julio de 1970).
} 


\subsection{La estrategia lobbista}

FUNSA vería con recelo cualquier incursión en el mercado de neumáticos y productos de goma, sea por contrabando, acuerdos, inversiones extranjeras o iniciativas nacionales y se comienza a activar un mecanismo de consulta y demanda permanente con el poder gobernante de turno.

En octubre de 1963 se denunciaba que habían ingresado en forma de contrabando desde el exterior en los últimos dos años alrededor del $16 \%$ del volumen de ventas de la empresa, lo cual significaba pérdidas considerables (FUNSA, Actas Asambleas de Accionistas, libro 2, Acta n. 34, octubre de 1963). En el mismo sentido, en 1965 se plantea una demanda al Ministerio de Hacienda por la existencia de vehículos con neumáticos importados en el puerto de Nueva Palmira (en la frontera con Argentina), violatorios del decreto del 29 de diciembre de 1964 (FUNSA, Actas Directorio, Acta n. 1.190, enero de 1965, p. 599).

En 1969, la empresa BENUSA de Estados Unidos, busca instalar una fábrica de recauchutaje de cascos importados lo que se ve con preocupación (FUNSA, Actas Directorio, Acta n. 1.337, marzo de 1969) y se iniciarán gestiones para detener su instalación (FUNSA, Actas Directorio, Acta n. 1.338, abril de 1969), proponiendo incluso legislar al respecto. De esta forma se instala una estrategia de "lobby" cada vez más explícita, definida como una serie de acciones concertadas para obtener beneficios de parte del gobierno. Veamos algunos ejemplos.

En 1967 se envia una nota al presidente de la República, Oscar Gestido, "por la eficaz actuación de las autoridades endefensa de los intereses uruguayos y de la industria de ALALC" ${ }^{4}$. Se contrata al Dr. Vargas para que gestione la derogación del 10\% de recargo de importación de materias primas. Ese año se mantienen reuniones con el ministro de Industria, Zelmar Michelini, y Dr. Massa, para ajustar un decreto del Poder Ejecutivo, y con la Oficina de Planeamiento y Presupuesto a cargo de Luis Faroppa acordando que las importaciones de

4 FUNSA, Actas Directorio, Acta n. 1.261, julio de 1967, p. 721. La Asociación Latinoamericana de Libre Comercio fue creada con el objetivo de reducir aranceles y aumentar los países firmantes del Tratado de Montevideo en 1960, posteriormente fue sustituida por ALADI; ver <http://www.aladi.org>, accedido el diciembre de 2017. 
Materias Primas para producción local tendria cambio asegurado dentro de los 60 días de despacho. Y con el Ministerio de Hacienda para discutir la situación del sector (FUNSA, Actas Directorio, Acta n. 1.266, 1967, p. 731).

Entre noviembre y diciembre de 1968 se mantendrán reuniones con el ministro del Interior, Aréchaga, por necesidades financieras y la rendición de cuentas que incluía un artículo referido a los autos en frontera (FUNSA, Actas Directorio, Acta n. 1.322, noviembre de 1968), con el ministro de Ganadería, Frick Davie, dado un acuerdo sobre importaciones arroceras que incluía cubiertas y debía ser modificado (FUNSA, Actas Directorio, Acta n. 1.325, diciembre de 1968), y con el subsecretario de Trabajo sobre los standards de calidad de bolsas de agua caliente (FUNSA, Actas Directorio, Acta n. 1.327, diciembre de 1968). Esta estrategia lobbista se profundizará con el gobierno de la dictadura.

La creación de la Comisión de Precios Productividad e Ingresos (COPRIN) por parte de la Ley n. 13.720, del 16 de diciembre de 1968, abrirá un nuevo periodo en materia de presiones sobre el poder político. Se analiza cómo afecta la producción de la empresa (FUNSA, Actas Directorio, Acta n. 1.296, mayo de 1968) y se plantean nuevas protecciones legales al neumático, que Hacienda desestima (FUNSA, Actas Directorio, Acta n. 1.316, setiembre de 1968), hay que recordar que ya existían beneficios como la exoneración de las actividades bancarias por las que se obtenían reintegros.

Entre 1969 y 1971 se producen infinidad de contactos con el Poder Ejecutivo (FUNSA, Actas Directorio, Acta n. 1.446, julio de 1971; n. 1.458, octubre de 1971), donde destaca la preocupación por la participación de empresas extranjeras en las licitaciones públicas, ello daría lugar al Decreto por del 21 de octubre de 1971 por el que se da preferencia y financiamiento en las licitaciones públicas a materiales y equipos nacionales.

Esta línea de apoyo a la industria tendría un nuevo hito en 1972, mediante la declaración de Interés Nacional el proyecto de ampliación de plantas de neumáticos, quedando exceptuados todo tributo o gravamen, aportes o préstamos nacionales (FUNSA, Actas Directorio, Acta n. 1.505, noviembre de 1972). La misma seguirá el gobierno de dictadura, que daría prioridad a FUNSA en los proyectos, "para salvaguardar intereses nacionales” (FUNSA, Actas Directorio, Acta n. 1.589, octubre de 1974). 


\subsection{Relacionamiento con los trabajadores en 1958-1974}

Este periodo es sumamente conflictivo dado la alta inflación y el estancamiento económico (Finch, 2014, p. 243-286).

En 1958 se produce una huelga de 100 días y como resultado se duplica el número de días de licencia pasando a 23 (FUNSA, Actas Asambleas de Accionistas, libro 2, Acta n. 28, setiembre de 1958). Al año siguiente se producen 70 días de huelga y se mantendrá un Convenio Colectivo hasta 1964 (FUNSA, Actas Asambleas de Accionistas, libro 2, Acta n. 31, setiembre de 1960), conflictos similares continuarán los años siguientes (FUNSA, Memoria y balance, 1959-1965).

El año 1965 sería de negociación, los trabajadores plantean el aumento del costo de vida y la renovación del Convenio de Seguridad y Productividad (FUNSA, Actas Directorio, Acta n. 1.163, junio de 1965), la caída del convenio produce que la jornada semanal se lleva a 60 horas (FUNSA, Actas Directorio, Acta n. 1.169, junio de 1965, p. 567). Si bien FUNSA tiene un promedio salarial mayor que el resto de la industria (según la empresa) en 1966 (FUNSA, Actas Directorio, Acta n. 1.191, 1966) se firma el nuevo convenio al igual que en 1967 donde el ajuste se calcula en 36,658\% (FUNSA, Actas Directorio, Acta n. 1.261, julio de 1967, p. 721). El año siguiente se aprueba una fórmula similar con el régimen semanal de 60 horas en cinco días (FUNSA, Actas Directorio, Acta n. 1.316, setiembre de 1968).

En junio de 1969 la empresa acuerda con el gobierno no enviar trabajadores al seguro de paro, para no afectar las finanzas el instituto jubilatorio, con el que además se mantiene una deuda existente (FUNSA, Actas Directorio, Acta n. 1.348, junio de 1969). Al mismo tiempo se lleva adelante una nueva negociación del Convenio Colectivo, acordado con COPRIN el aumento de precios, se aprueba un $8 \%$ con aumento de productividad a cambio de la posibilidades de extender préstamos a los trabajadores (FUNSA, Actas Directorio, Acta n. 1.355, julio de 1969; n. 1.366, octubre de 1969).

En 1970, la empresa cuenta con 1976 empleados (FUNSA, Actas Directorio, Acta n. 1.391, mayo de 1970), hasta 1974 se produce un fenómeno permanente, las negociaciones salariales estarán signadas por la relación con los precios, dado que estos eran fijados por el gobierno. El balance de 1972 representa un buen ejemplo: 
Durante el ejercicio terminado se han operado por disposición del gobierno dos ajustes salariales en octubre de 1971 (27.2\% y en abril de 1972 20\%). Por diversos motivos, este periodo se ha caracterizado por la inestabilidad laboral en el ámbito nacional. En nuestro caso, junto a múltiples interrupciones de trabajo de corta duración, se produjeron dos paralizaciones de entidad, abarcando la primera de ellas del 20 de agosto al 15 de setiembre de 1971 y la segunda del 22 de junio al 10 de julio de 1972. (FUNSA, Memoria y balance, 1972)

La planta se vería ocupada por el conflicto político, y también por razones salariales en todo este periodo. La planta ocupada del 5 al 7 de setiembre en 1972 solicitando 35\% de aumento de salarios, y un mes y medio por motivo del golpe de estado en 1973, tras lo cual se reanudarán los trabajos, con préstamos y adelantos al personal a cuenta de elevar la producción a 1.100 cubiertas diarias (FUNSA, Memoria y balance, 1973).

\subsection{La estrategia exportadora}

En este periodo se intensificaron las relaciones comerciales con la región, primero con Argentina y Brasil, y en menor medida con Paraguay casi simbólica (FUNSA, Actas Directorio, Acta n. 1.488, n. 1.489, junio de 1972; n. 1.497, n. 1.498, octubre de 1972; n. 1.530, 27 de junio de 1973; n. 1.531, n. 1.532, julio de 1973).

Si bien desde fines de 1950 ya habían comenzado a exportar a Argentina, hacia 1967 surgen las primeras trabas por especificaciones de origen realizando diferentes gestiones para levantar las mismas, a modo de ejemplo en marzo de 1968, la deuda por productos exportados ese mes ascendió a 316 mil dólares (FUNSA, Actas Directorio, Acta n. 1.281, diciembre de 1967; n. 1.300, junio de 1968, p. 797).

La apertura del mercado de Brasil supuso una serie de gestiones a nivel privado y político. Por un lado se firma con convenio de distribución con la empresa Coral SA. realizado en octubre de 1968, animado por el embajador de Brasil, Domingo del Campo, firmando una cuota de exportación de 30.000 cámaras y comenzando con una primer partida de 118 mil dólares en abril de 1969. La cuota se duplicaría rápidamente a 90.000 cámaras anuales (FUNSA, Actas Directorio, Acta n. 1.327, 
n. 1.329 , diciembre de 1968; n. 1.334, marzo de 1969; n. 1.347, junio de 1969).

La empresa solicitará al gobierno apoyo para sostener estas exportaciones: reducción de la tasa de interés, apoyo crediticio, desgravación del costo del dólar industrial, préstamos de largo pazo, defensa del mercado interno, equilibrio y acuerdo en materia internacional. Este periodo no estará exento de dificultades en las exportaciones a Brasil y Argentina, pero parece evidente que las mismas jugaron un rol fundamental en tanto diversificar las posibilidades productivas de la empresa, en ambos casos se buscarán acuerdo de complementación productiva, lleva las ventas a 10 millones de dólares anuales (FUNSA, Actas Directorio, Acta n. 1.544, octubre de 1973).

\section{Primeras conclusiones}

A los efectos de nuestro trabajo resulta importante analizar el rol del Estado uruguayo en la estructura de incentivos rentistas que establece la legislación, así como la existencia de una ideología que podemos denominar proteccionista, basada en la preeminencia del neobatllismo ${ }^{5}$, la política de industrializacion por sustitución de importaciones y su agotamiento como modelo a principios de los 60. Parece que la existencia en tanto surgimiento, auge y desarrollo de la empresa FUNSA está íntimamente ligada a la legislación y las política de incentivos y a las instituciones (en sentido northiano), surgidos desde el Estado que se articula con una ideología proteccionista e interventora.

En segundo lugar, luego de considerar las diferentes fuentes disponibles, las estimaciones de Ocupación,VAB, del Sector Caucho y FUNSA, parece claro que el peso de FUNSA en el mercado uruguayo en el periodo estudiado puede ser catalogado de monopólico, sin embargo, una revisión de las estimaciones y triangulación de fuentes se hace necesaria para robustecer dicha hipótesis, fundamentalmente considerando los criterios para definir el número de establecimientos en competencia así como la ocupación del sector (Gráfico 2). En lo salarial confirmamos

5 Se denomina así al período signado por el desarrollo de políticas industriales y económicas dirigistas del Estado (Finch, 2014). 
mediante análisis comparativo nuestras primeras estimaciones y a su vez el peso de los Consejos de Salarios y la organización sindical que operan aumentos notables una vez que los mismos comienzan a operar como mecanismos de fijación de salarios.

Parece que FUNSA, comparte una serie de características de la "modern enterprise" de Chandler: directores altamente especializados en sus tareas con un creciente dominio del mercado local, hasta conformar un monopolio, así como las búsquedas de expansión hacia mercados regionales. A su vez, combina el predominio de mercado de la industria moderna, con una tecnología superior en lo local, masificación, gigantismo y la renta derivada de las protecciones que derribaban de la ley y su consecuente apropiación de la renta nacional.

Por otra parte tampoco hay dudas de la división de propiedad y gestión en FUNSA en su etapa fundacional, sin embargo se identifica un proceso de apropiación de parte de los principales directores del capital accionario, lo cual responde a un proceso que lleva varias décadas, a partir de una posición preferencial de los directivos que casi no cambian en todo el periodo estudiado.

Como se ha visto, una de las líneas de larga duración refiere al asesoramiento técnico que FUNSA recibió en todo el periodo, retomando a Zeitlin y Sabel (1997) podríamos decir que la empresa explotó al máximo sus redes empresariales y políticas para adaptarse a las necesidades del mercado y ocupar un lugar de relevancia, en primer lugar con BF Goodrich (1936-1946) quien colaboró con la instalación de la planta y las primeras producciones de neumáticos, en esa línea principal sería retomada por U.S. Rubber en 1945-1960, y por esa época para la producción de cámaras y cubiertas comenzará también una prolongada relación con la italiana Pirelli. Desde 1960 en adelante la preocupación por la tecnología y la modernización se plasmará en un potente acuerdo con Firestone.Así como las estrategias de captación de capital que supo pasar del financiamiento local a bancario nacional e internacional. En el mismo sentido debe apuntarse la explotación de redes políticas, como se vio a lo largo de la búsqueda de materias primas, estrategia lobbista y la captación de financiamiento bancario.

Por último, la idea de observar la evolución de la industria en el uruguay a través de un estudio de caso, parece ajustarse en esta ocasión, en la medida que el relato de la evolución industrial del Uruguay, con 
dos periodos marcados por el crecimiento explosivo entre 1930-1958, seguido del estancamiento en 1960 y repunte al final de esa década, se observa en FUNSA.

\section{Fuentes}

\section{Fuentes primarias}

FUNSA - Fábrica Uruguaya de Neumáticos Sociedad Anónima. Actas Asambleas de Accionistas. Montevideo, libro 1, 1936-1947.

FUNSA - Fábrica Uruguaya de Neumáticos Sociedad Anónima. Actas Asambleas de Accionistas. Montevideo, libro 2, 1948-1963.

FUNSA - Fábrica Uruguaya de Neumáticos Sociedad Anónima. Actas Asambleas de Accionistas, tinta a pluma. Montevideo, 1941-1950.

FUNSA - Fábrica Uruguaya de Neumáticos Sociedad Anónima. Actas Directorio, copia en carbónico. Montevideo, n. 911-1.016, 1959-1962;n.1.017-1.084,1962;n.1.0851.148,1963-1965;n.1.105-1.156,1965;n. 1.157-1.224,1965-1966;n.1.245-1.440, 1966-1971;n.1.324-1.439,1966-1971;n.1.441-1.554,1971-1973;n.1.555-1.606, 1974-1975.

FUNSA - Fábrica Uruguaya de Neumáticos Sociedad Anónima. Libro de egresos, tinta. Montevideo, 1938-1949.

FUNSA - Fábrica Uruguaya de Neumáticos Sociedad Anónima. Libro de egresos, tinta. Montevideo, 1950-1959.

FUNSA - Fábrica Uruguaya de Neumáticos Sociedad Anónima. Memoria y balance. Montevideo, 1936-1974.

\section{Prensa}

1825-25 DE AGOSTO DE 1940, 115 años de vida independiente, Fábrica Uruguaya de Neumáticos S.A. El Pueblo, s.p., 25 de agosto de 1940.

ANÁLISIS del Triunfo de los Trabajadores de FUNSA. El Sol. Montevideo, p. 2, 30 de diciembre de 1952.

CAGGIANI, Ruben. La convocatoria de los Consejos de Salarios. El Sol. Montevideo, 4 de agosto de 1954.

DESPOTISMO en FUNSA. El Sol. Montevideo, p. 2,11 de mayo de 1955.

DOS REPROCHES se han dirigido a FUNSA. El País, s.p., 16 de febrero de 1946.

FUNSA. El País, p. 43, 11 de julio de 1943.

F.U.N.S.A, conviene que se sepa como se trata de justificar una política de paros diarios. El Debate. Montevideo, p. 2, 15 de diciembre de 1956. 
F.U.N.S.A, los obreros rechazan una fórmula que les asigna los salarios más altos de la industria. Acción. Montevideo, p. 9, 12 de julio de 1957.

GRAVES cargos contra FUNSA. El Sol. Montevideo, p. 2, junio de 1955.

HECHO que congratula: culminación de anhelos del sindicato de FUNSA. El Día. Montevideo, 11 de noviembre de 1962.

LOCKOUT en FUNSA: una empresa multimillonaria que pretende imponer sus caprichos. El Sol. Montevideo, 24 de abril de 1955.

LOS OBREROS de FUNSA frenan a la patronal. El Sol. Montevideo, 9 de julio de 1954.

NUEVAS acciones contra FUNSA, prepotencia patronal y tolerancia cómplice del gobierno. El Sol. Montevideo, p. 2, 9 de diciembre de 1952.

PRE-CONFLICTO en FUNSA, arbitrariedades de la empresa agravan la situación. El Sol. Montevideo, p. 2, 20 de enero de 1953.

RECRUDECE el despotismo en FUNSA. El Sol. Montevideo, p. 4, 20 de enero de 1954.

SÁENZ, Pedro. F.U.N.S.A a la opinión pública, un nuevo esfuerzo de pacificación que no obtuvo respuesta de la parte obrera. El Debate. Montevideo, 19 de diciembre de 1956.

SOBRE la huelga en FUNSA. El Sol. Montevideo, 23 de noviembre de 1956.

SOLIDARIDAD con los obreros de FUNSA. El Sol. Montevideo, 26 de octubre de 1956.

UNA EMPRESA multimillonaria que explota a sus trabajadores. El Sol. Montevideo, p. 2, 4 de noviembre de 1952.

UN FEUDO a la moderna. El Sol. Montevideo, 12 de octubre de 1956.

WILLIMAN, José Claudio (h). El conflicto periódico de FUNSA. Marcha. Montevideo, 28 de agosto de 1953.

\section{Referencias bibliográficas}

AGUIRRE, Gustavo; DELLEPIANE, Adrián; DUARTE, Horacio. Aspectos de la estructura y producción de neumáticos en el Uruguay: el caso FUNSA. Montevideo: Universidad de la República, 2004 (Tesis de Grado).

ARNABAL, Luis Rodrigo; BERTINO, Magdalena; FLEITAS, Sebastián. Una revisión del desempeño de la industria en Uruguay entre 1930 y 1959. Revista de Historia Industrial, año XXI, n. 53, 2013. Disponible en < http://www.raco.cat/index.php/ HistoriaIndustrial/article/view/271341/358996>. Accedido el agosto de 2017.

AZAR, Paola; FLEITAS, Sebastián. Las relaciones entre Estados Unidos y Uruguay en el largo plazo. Montevideo: Instituto de Economía, 2010 (Serie Documentos de Trabajo, DT 1/10).

BCU - Banco Central del Uruguay. Cuentas nacionales. Montevideo, 1965. 
BCU - Banco Central del Uruguay. Cuentas nacionales. Montevideo: Departamento de Investigaciones Económicas, 1969.

BCU - Banco Central del Uruguay. Producto e ingreso nacionales. Montevideo, 1989.

BÉRTOLA, Luis. La industria manufacturera uruguaya. Un enfoque sectorial de su crecimiento, fluctuaciones y crisis. Montevideo: Facultad de Ciencias Sociales/CIEDUR, 1991.

BÉRTOLA, Luis; BITTENCOURT, Gustavo. Un balance histórico de la industria uruguaya: entre el "destino manifiesto" y el voluntarismo. Montevideo: MIEM-DNI/Facultad de Ciencias Sociales/UDELAR, 2014.

BÉRTOLA, Luis et al. Southern cone real wages compared: a purchasing power parity approach to convergence and divergence trends, 1870-1996. XXVII ENCONTRO NACIONAL DE ECONOMÍA, ANPEC, 1999, Belém, p. 545-569.

BROU - Banco de la República. Cuentas nacionales. Montevideo, 1965.

CABALLERO, G. Instituciones e historia económica: enfoques y teorías institucionales. Revista de Economía Institucional. España, v. 6, n. 10, 2004.

CABRAL, Ruth; CHACHARO, Luciano; MISEVICIUS, Virginia. Análisis estratégico de una organización que reabre bajo una nueva forma de gestión. Montevideo: Facultad de Ciencias Económicas y Administración, Universidad de la República, 2006 (Tesis de Grado).

CGN - Contaduría General de la Nación. Boletín de Hacienda. Montevideo, año XXVIII, n. 1-6, 1941.

CHANDLER, Alfred D., Jr. Scale and scope: the dynamycs of industrial capistalism. London/Cambridge (Mass.):The Belknap Press of Harvard University Press, 1990.

CHEVALIER, Mariángeles; FERNÁNDEZ, Claudio; MOTTA,Virginia. Los Consejos de Salarios en el Uruguay, 1943-1950. Montevideo: Facultad de Ciencias Económicas y Administración, Universidad de la República, 2006 (Tesis de Grado).

DÍAZ CASERO J.; URBANO PULIDO, D.; HERNÁNDEZ MOGOLLÓN, R. Teoría económica institucional y creación de empresas. Barcelona: Investigaciones Europeas de Dirección y Economía de la Empresa (IEDEE), 2005.

FCS - Facultad de Ciencias Sociales. Banco de datos. Montevideo: Universidad de la República, 2005.

FERNÁNDEZ CAETANO, Claudio. Los Consejos de Salarios en el Uruguay en la década del cuarenta. Su contexto y resultados. Los casos de la industria gráfica, del caucho, del cuero y química. H-Industria, Revista de Historia de la Industria, los Servicios y las Empresas en América Latina, año 6, n. 11, segundo semestre 2012.

FINCH, Henry. La economía política del Uruguay contemporáneo, 1870-2000. Montevideo: Colección Heber Raviolo, 2014 (1. ed. 1980, corr. y aum. 2005).

GONZÁLEZ SIERRA, Yamandú. Un sindicato con historia. Tomo I, II. Montevideo: Unión de Obreros, Empleados y Supervisores de FUNSA/Federación de Caucho/ PIT-CNT, 1991.

GONZÁLEZ SIERRA,Yamandú. Un sindicato con historia.Tomo III. Montevideo: Unión de Obreros, Empleados y Supervisores de FUNSA/Federación de Caucho/PITCNT, 1998. 
IECON - Instituto de Economía. Estadísticas básicas Uruguay. Montevideo: Facultad de Ciencias Económicas y Administración, Universidad de la República, 1969.

JACOB, Raúl. Breve historia de la industria en el Uruguay. Montevideo: Fundación de Cultura Universitaria, 1981.

JACOB, Raúl. Banca e industria: un puente inconcluso. Montevideo: Fundación de Cultura Universitaria/CIEDUR, 1991.

JACOB, Raúl. La quimera y el oro. Montevideo: Arpoador, 2000.

JACOB, Raúl. La historia de empresas en Uruguay en BARBERO, María Inés; JACOB, Raúl. La nueva historia de empresas en América Latina y España. Buenos Aires: Temas, 2008.

JACOB, Raúl. Las inversiones norteamericanas, 1900-1945. Montevideo: Objeto Directo, 2016.

MILLOT, Julio; SILVA, Carlos; SILVA, Lindor. El desarrollo industrial del Uruguay de la Crisis de 1929 a la posguerra. Montevideo: Instituto de Economía/Universidad de la República, 1973.

NORTH, Douglass C. Institutions, institutional change, and economic performance. Cambridge/New York: Cambridge University Press, 1990.

ODDONE, Juan. Uruguay en los años 30. Montevideo: FCU, 1989 (Cuadernos de Interguerras).

OFICIALDEGUI, Marta. Las relaciones comerciales triangulares uruguayas entre 1909 y 1953: una aproximación a su estudio. Montevideo: Programa de Historia Económica y Social, Facultad de Ciencias Sociales, UDELAR, 1995.

RODRÍGUEZ PLÁ, Américo. Antecedentes inmediatos de la Ley 10.449, en los Consejos de Salarios una mirada actual, $A A$. VV. Montevideo: Fundación de Cultura Universitaria, Cuadernos de Fundación, Derecho del Trabajo y la Seguridad Social, 2004.

SARTHOU, Helios. Rasgos principales del régimen de los Consejos de Salarios creados por la Ley 10.449, en los Consejos de Salarios una mirada actual, AA. VV. Montevideo: Fundación de Cultura Universitaria, Cuadernos de Fundación, Derecho del Trabajo y la Seguridad Social, 2004.

TORRESVILLANUEVA, Eugenio. Funciones empresariales, cambio institucional $y$ desarrollo económico en DÁVILA L. DE GUEVARA, Carlos. Empresas y empresarios en la historia de Colombia. Siglos XIX y XX. Bogotá: Facultad de Administración/ CEPAL, 2002.

ZEITLIN, Jonathan; SABEL, Charles. World of possibilities. Flexibility and mass production in Western industrialization. Cambridge: Maison des Sciences de l'Homme/ Cambridge University Press, 1997. 